\title{
Revisión de 25 casos de papiloma invertido en 8 años
}

\author{
Review of 25 cases of inverted papilloma in 8 years
}

Jaime Osorio $\mathbf{M}^{1}$, Lorena Aguayo G'1 , Pilar Gajardo $0^{1}$.

\begin{abstract}
RESUMEN
Introducción: El papiloma invertido es un tumor benigno inusual del tracto nasosinusal, caracterizado por el riesgo de recurrencia y transformación maligna.

Objetivo: Nuestro objetivo es describir la serie de casos diagnosticados con papiloma invertido del tracto nasosinusal entre 2002 y 2010.

Material y método: Se trata de un análisis retrospectivo de los casos nuevos de papiloma invertido del tracto nasosinusal atendidos en nuestro Hospital entre los años 2002 y 2010.

Resultados: Se presentaron 25 casos nuevos de papiloma invertido. Los síntomas tenían en promedio 2 años de evolución, siendo la obstrucción nasal y rinorrea los más frecuentes. La etapificación preoperatoria fue realizada mediante tomografía computarizada en 15 pacientes. El abordaje endoscópico fue el preferido. Cincuenta y seis por ciento de los pacientes correspondía a la etapa 2 de la clasificación de Krause. El promedio de seguimiento fue 29 meses. Durante ese periodo, 4 casos presentaron recurrencia, correspondiendo al $16 \%$ de nuestra serie. La mitad de ellos presentó displasia o atipia al momento de la recurrencia. Ocurrió 1 caso de transformación maligna, el cual se presentó como un carcinoma indiferenciado.

Conclusión: Las características generales son similares a los reportes internacionales. En nuestro medio los pacientes son diagnosticados en etapas avanzadas de la enfermedad, no obstante, el abordaje endoscópico es el método quirúrgico preferido.
\end{abstract}

\begin{abstract}
Introduction: Inverted papilloma is an unusual benign tumor of the sinonasal tract, characterized by an important risk of recurrence and malignant transformation.

Aim: Our objective is to describe the case series diagnosed with inverted papilloma of the sinonasal tract between the years 2002 and 2010.

Material and method: A retrospective analysis of new cases of inverted papilloma of the sinonasal tract treated at our Hospital between the years 2002 and 2010.

Results: 25 cases of newly diagnosed inverted papilloma presented in our institution. The symptoms had on average 2 years of evolution, being nasal obstruction and rhinorrhea the most frequent. Preoperative staging by computed tomography (CT) was performed in 15 patients. The endoscopic aproach was preferred as surgical approach. $56 \%$ of the patients corresponded to stage 2 of Krause classification. Median folow up was 29 months. During that period, 4 patients had recurrence, corresponding to $16 \%$ in our series. Half of these patients had dysplasia or atypia at the time of recurrence. 1 case of malignant transformation ocurred, which presented as an undifferentiated carcinoma.

Conclusions: General characteristics were similar to those reported in international reports. In our setting, patients are diagnosed in advanced stages of disease, nevertheless, endoscopic approach is the preferred surgery method.
\end{abstract}

${ }^{1}$ Médicos del Servicio de Otorrinolaringología, Hospital Barros Luco Trudeau. 


\section{INTRODUCCIÓN}

El papiloma invertido es una neoplasia poco frecuente de la región nasosinusal, caracterizada por su ubicación unilateral, invasión multicéntrica, tendencia a la recidiva y hasta $10 \%$ de malignización. Se presenta habitualmente entre la quinta y sexta década de la vida y es más frecuente en el sexo masculino. En términos histológicos, presentan un crecimiento endofítico con cambios polipoides de la mucosa nasal, con metaplasia escamosa ${ }^{1,2}$.

El tratamiento clásico del papiloma invertido era la resección en bloque mediante rinotomía lateral o maxilectomía media a través de una incisión abierta ${ }^{3}$. Con el advenimiento de las técnicas quirúrgicas endoscópicas de los senos paranasales, cirujanos experimentados comenzaron a utilizarlas en papiloma invertido en forma progresiva, con buenos resultados, igualando las cifras de recurrencia, encontrando experiencias positivas también en nuestro país ${ }^{4-6}$.

Considerando la necesidad de contar con un mecanismo para evaluar la efectividad de los diferentes abordajes, Krouse ${ }^{4}$ estableció un sistema de etapificación basado en la localización, extensión y malignidad de las lesiones, establecida por tomografía computarizada (TC) y endoscopía (Tabla 1). Más tarde surgieron nuevas clasificaciones, tales como $\mathrm{Han}^{7}$, Cannady ${ }^{8}$ y Dragonetti-Minni, quienes proponen en 2011 una nueva clasificación ${ }^{9}$. Ninguna de ellas ha sido adoptada en forma generalizada.

Algunos autores han señalado que existe asociación entre las características histológicas del tumor, particularmente displasia, y la aparición de recidivas 0 transformación maligna ${ }^{10}$.

\section{OBJETIVO}

Nuestro objetivo es describir la serie de casos diagnosticados con papiloma invertido del tracto nasosinusal entre los años 2002 y 2010 en nuestro centro clínico docente, el Hospital Barros Luco Trudeau, con énfasis en las características generales de los casos detectados, clasificación de Krouse y concordancia con hallazgos quirúrgicos, vía de resolución quirúrgica, porcentaje de recurrencias y su relación con atipia o displasia y porcentaje de malignización.
Tabla 1. Sistema de etapificación para papiloma invertido, según Krouse

\begin{tabular}{|c|c|}
\hline & Descripción \\
\hline T1 & $\begin{array}{l}\text { Tumor totalmente confinado a la cavidad nasal, } \\
\text { sin extensión dentro de los senos. El tumor } \\
\text { puede estar localizado en una pared o región } \\
\text { de la cavidad nasal, o puede ser voluminoso } \\
\text { y extenso dentro de la cavidad nasal, pero no } \\
\text { debe extenderse dentro de los senos o dentro de } \\
\text { cualquier compartimiento extranasal. No debe haber } \\
\text { malignidad concurrente. }\end{array}$ \\
\hline T2 & $\begin{array}{l}\text { Tumor compromete el complejo ostiomeatal, seno } \\
\text { etmoidal, y/o la porción medial del seno maxilar, } \\
\text { con o sin compromiso de la cavidad nasal. No debe } \\
\text { haber malignidad concurrente. }\end{array}$ \\
\hline T3 & $\begin{array}{l}\text { Tumor compromete las paredes lateral, inferior, } \\
\text { superior, anterior o posterior del seno maxilar, } \\
\text { el seno esfenoidal, y/o el seno frontal, con o sin } \\
\text { compromiso de la porción medial del seno maxilar, } \\
\text { seno etmoidal o la cavidad nasal. No debe haber } \\
\text { malignidad concurrente. }\end{array}$ \\
\hline T4 & $\begin{array}{l}\text { Todos los tumores con cualquier extensión } \\
\text { extranasal/extrasinusal que comprometa estructuras } \\
\text { adyacentes, contiguas, tales como la órbita, } \\
\text { el compartimiento intracraneal, o el espacio } \\
\text { pterigomaxilar. Todos los tumores asociados a } \\
\text { malignidad. }\end{array}$ \\
\hline
\end{tabular}

\section{MATERIAL Y MÉTODO}

Se realizó un estudio retrospectivo de los casos de papiloma invertido nasosinusal diagnosticados entre Ios años 2002 y 2010 según los registros del Servicio de Anatomía Patológica de nuestro hospital. Se identificaron edad, sexo, síntomas y tiempo de evolución de los mismos. Se determinó la extensión de las lesiones por TC, clasificándolas según Krouse. Además se evaluó la vía de resolución quirúrgica y la clasificación obtenida según la descripción del protocolo operatorio. Finalmente se analizaron los casos de recidiva.

\section{RESULTADOS}

Se identificaron 25 casos nuevos de papiloma invertido nasosinusal. El $63 \%$ de la muestra era de sexo masculino y el promedio de edad fue 57 años. El tiempo de evolución de los síntomas fue en promedio 2 años, con un rango entre 3 meses y 6 años.

Dentro de los síntomas de presentación, el más frecuente fue obstrucción nasal, seguida en orden 
decreciente por rinorrea, masa en fosa nasal, cefalea, epistaxis y dolor (Figura 1). En forma aislada se presentaron anosmia y roncopatía.

Se tuvo acceso al informe de TC realizada en forma preoperatoria de quince pacientes, los cuales fueron clasificados según Krouse. No se realizó resonancia nuclear magnética (RNM) preoperatoria a ningún paciente de nuestra serie. Se comparó dicha clasificación con la obtenida posterior a la cirugía, según lo descrito en el protocolo operatorio (Figura 2). Evaluamos la concordancia entre la clasificación preoperatoria mediante TC y la posoperatoria según los hallazgos quirúrgicos mediante el gráfico de Bland Altman. La distribución de las diferencias entre las variables no mostró ninguna tendencia que sugiera sesgo, existiendo además diferencias iguales a cero, por lo que la evaluación preoperatoria en estos 15 pacientes tuvo una concordancia aceptable con respecto al hallazgo quirúrgico (Figura 3). Evaluando la clasificación caso a caso, existió una sobreestimación de la extensión tumoral en cinco pacientes y una subestimación en un paciente mediante la evaluación por TC.

La clasificación según el hallazgo quirúrgico para el total de pacientes se puede observar en la Figura 4.
Respecto a la resolución quirúrgica, ésta fue por vía degloving mediofacial o rinotomía lateral en el $16 \%$ de los casos, mientras que en el $80 \%$ fue endoscópica. Un paciente no fue sometido a cirugía. El tiempo transcurrido entre el diagnóstico y la cirugía fue en promedio 7,7 meses.

El tiempo de seguimiento de los pacientes desde la resolución quirúrgica hasta el último control médico fue en promedio 29 meses, con un rango entre 0 y 105 meses.

Se confirmaron cuatro pacientes con recidiva, cuyas características se resumen en la Tabla 2. La paciente $\mathrm{N}^{\circ} 2$ presentó una segunda recidiva de localización etmoidal, 39 meses después de su segunda cirugía. La resolución fue vía endoscópica, sin nuevas recidivas hasta la fecha. Los pacientes que recidivaron no presentaron en la biopsia inicial displasia ni atipia, sin embargo, estas características estuvieron presentes en dos pacientes en la cirugía de la recidiva. Respecto a la etapificación, los pacientes que recidivaron fueron clasificados inicialmente como T2 en el $50 \%$ y $\mathrm{T} 3$ en el otro $50 \%$. Los que no recidivaron fueron clasificados como T2 en el $66,6 \%$, T3 en $27,7 \%$ y T4 en $5,5 \%$. Un paciente se encuentra en estudio por sospecha de recidiva y su clasificación inicial fue T4.

Figura 1. Síntomas declarados por los pacientes al momento de la primera consulta otorrinolaringológica.

Figura 2. Comparación de la clasificación preoperatoria mediante TC y la clasificación posoperatoria según el hallazgo quirúrgico de 15 pacientes, según la clasificación de Krouse.
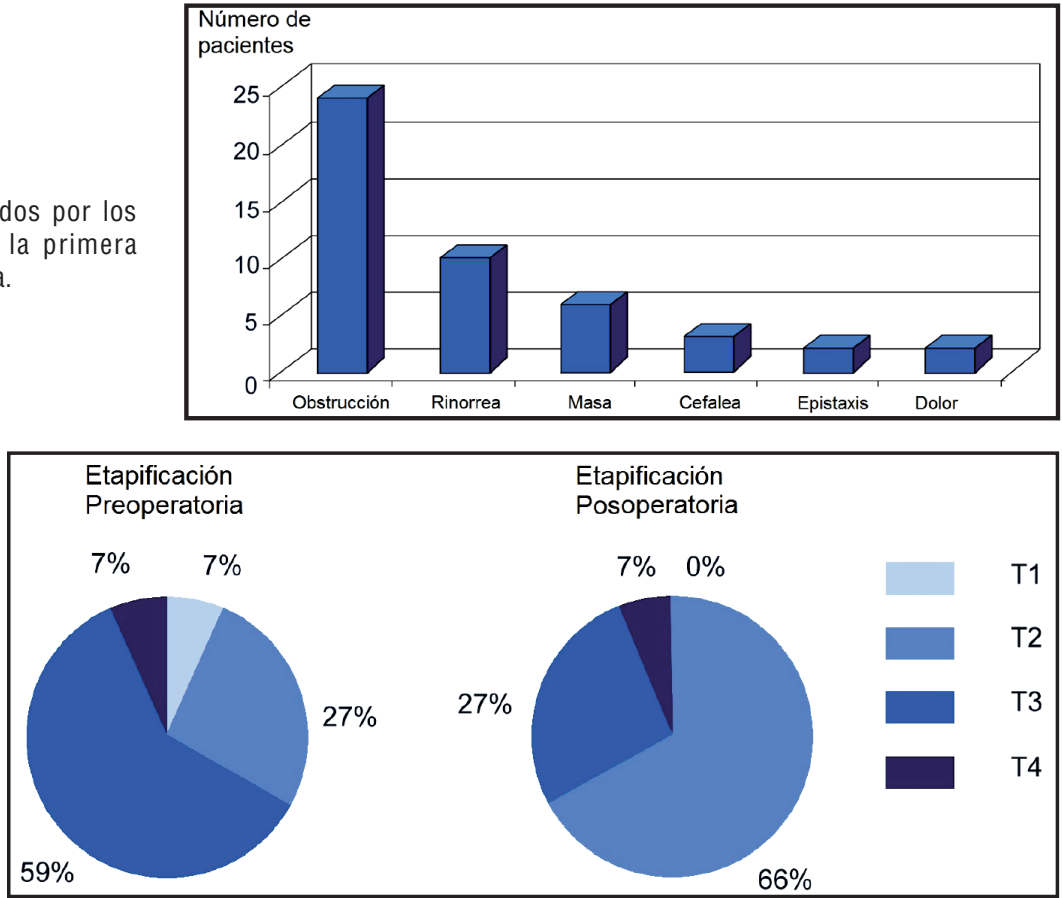
Tabla 2. Características de los pacientes que presentaron recidiva

\begin{tabular}{|c|c|c|c|c|c|}
\hline Paciente & $\begin{array}{c}\text { Localización } \\
\text { inicial }\end{array}$ & Vía de resolución & Tiempo en meses & Localización recidiva & Vía de resolución \\
\hline 1 & $\begin{array}{c}\text { Pared medial del } \\
\text { seno maxilar }\end{array}$ & Endoscópica & 13 & $\begin{array}{c}\text { Cavidad nasal, } \\
\text { seno maxilar }\end{array}$ & Enierta \\
\hline 2 & $\begin{array}{c}\text { Cavidad nasal, } \\
\text { seno maxilar }\end{array}$ & Endoscópica & 4 & Etmoides anterior & Abierta \\
\hline 3 & $\begin{array}{c}\text { Cavidad nasal, } \\
\text { etmoides, } \\
\text { pared medial } \\
\text { seno maxilar, } \\
\text { esfenoides }\end{array}$ & Endoscópica & 36 & $\begin{array}{c}\text { Cavidad nasal, paredes anterior } \\
\text { y medial del seno maxilar, } \\
\text { etmoides }\end{array}$ & Abierta \\
\hline 4 & $\begin{array}{c}\text { Cavidad nasal, } \\
\text { pared medial } \\
\text { seno maxilar }\end{array}$ & Endoscópica & 64 & Seno maxilar & \\
\hline
\end{tabular}

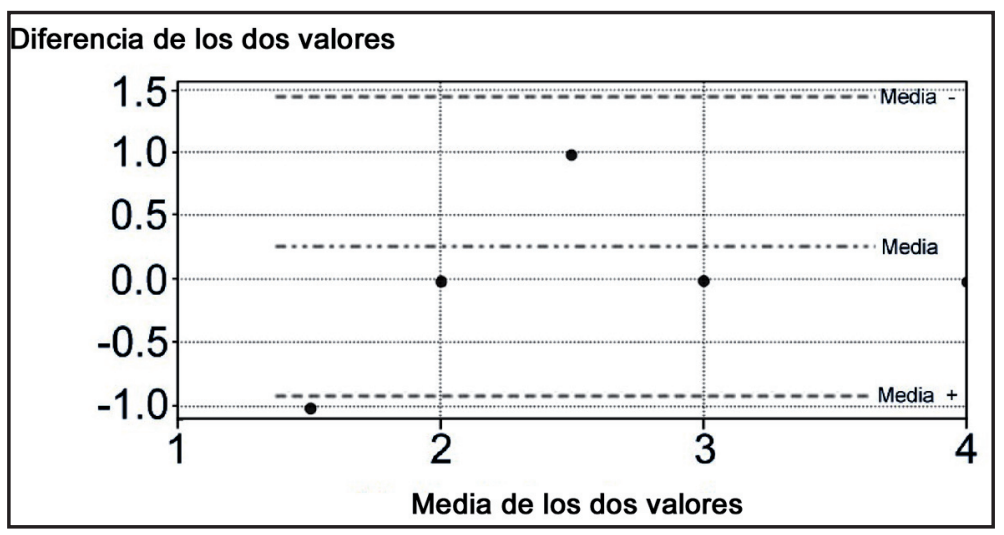

Figura 3. Gráfico de Bland Altman para evaluar concordancia entre la clasificación preoperatoria mediante TC y la clasificación posoperatoria según hallazgo quirúrgico, según la clasificación de Krouse, para 15 pacientes con papiloma invertido.

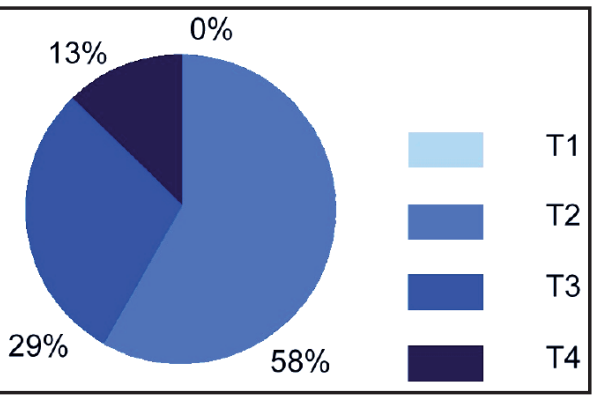

Figura 4. Clasificación de Krouse de todos los pacientes según hallazgo quirúrgico.

\section{DISCUSIÓN}

Las características generales de la muestra son similares a las reportadas en la literatura. La malignización presente en un paciente (4\% de la muestra), es menor a lo esperado, lo cual puede explicarse por el tiempo de seguimiento. Por lo tanto es posible que con el transcurso de los años se presenten uno o dos casos más de transformación maligna, lo que hace fundamental el seguimiento.

La clasificación preoperatoria mediante TC es un método aceptable. La sobreestimación de la extensión de las lesiones que ocurre en algunos casos se explica ya que este método no diferencia adecuadamente tumor de mucosa inflamada u ocupación líquida de los senos. Bhalla y Wright ${ }^{11}$ identificaron a la osteítis como un buen signo imagenológico a la TC para identificar la zona de inserción del tumor. Por otra parte, Karkos y cols ${ }^{12}$ llevaron a cabo una revisión sistemática centrándose en la evaluación radiológica del papiloma invertido. En los estudios incluidos se utilizaba TC 
la mayoría de las veces. La utilización de RNM en T2 era capaz de diferenciar tumor de tejido inflamatorio, sin embargo no se encontraron estudios que compararan la sensibilidad y especificidad del TC y RNM en la evaluación preoperatoria de papiloma invertido nasosinusal. La recomendación general actual es la utilización de TC y RNM combinadas.

Las recidivas en la literatura se presentan en una frecuencia muy variable. En nuestra serie correspondieron al $16 \%$ de los pacientes. Según el metaanálisis realizado por Busquets y $\operatorname{cols}^{13}$ en 2006, la resolución vía endoscópica presenta menor porcentaje de recidiva que aquella por abordaje abierto, similar al porcentaje encontrado en nuestra serie.

Ningún paciente presentó displasia en la primera resección quirúrgica. Sí se observó mayor frecuencia de pacientes etapa T3 (50\%).

Llama la atención la presencia inicial de compromiso del seno maxilar, especialmente de la pared medial en los pacientes que presentaron recidiva, presentando ésta en la misma localización, lo que podría apoyar la teoría de algunos autores, quienes refieren que en papiloma invertido lo que realmente ocurre es persistencia por resecciones incompletas.

\section{CONCLUSIÓN}

La clasificación del papiloma invertido sigue siendo un tema controversial y ninguna clasificación es utilizada en forma universal. Es recomendable utilizar tanto TC como RNM para la evaluación preoperatoria de estos pacientes.

La vía de resolución de elección actual es la endoscópica, ya que presenta menores porcentajes de recidiva.

Las recurrencias se presentan en tiempos muy variables, lo que sumado a la probabilidad de malignización exige tiempos de seguimiento prolongado.

\section{BIBLIOGRAFÍA}

1. Díaz JP, Llorente Jl, Rodrigo JP, Alvarez C, Obeso $\mathrm{S}$, SuÁrez C. Papilomas invertidos rinosinusales. Revisión de 61 casos. Acta Otorrinolaringol Esp 2009; 60: 402-8.
2. VRABEC DP. The inverted schneiderian papilloma: $A$ 25-year study. Laryngoscope 1994; 104: 582-605.

3. Lawson W, Ho BT, ShaAri CM, Biller HF. Inverted papilloma: A report of 112 cases. Laryngoscope 1995; 105: 282-8.

4. KROUSE JH. Development of a staging system for inverted papilloma. Laryngoscope 2000; 110: 965-8.

5. Wormald PJ, Ool E, Van Hasselt CA, Nair S. Endoscopic Removal of Sinonasal Inverted Papilloma Including Endoscopic Medial Maxillectomy. Laryngoscope 2003; 113: 867-73.

6. Sarmiento DH. Maxilectomía medial más etmoidectomía total endoscópica para el tratamiento del papiloma invertido nasosinusal. Rev Otorrinolaringol Cir Cabeza Cuello 2004; 64: 134-40.

7. Han JK, Smith TL, Loehrl T, et al. An evolution of the management of sinonasal inverting papilloma. Laryngoscope 2001; 111: 1395-400.

8. Cannady SB, Batra PS, Sautter NB, Roh HJ, Citardi MJ. New staging system for sinonasal inverted papilloma in the endoscopic era. Laryngoscope 2007; 117: 1283-7.

9. Dragonetti A, Gera R, Sciuto A, Scotti A, Barbaro E, Mınн A. Sinonasal inverted papilloma: 84 patients treated by endoscopy and proposal for a new classification. Rhinology 2001; 49: 20713.

10. Oikawa K, Furuta Y, Itoh T, Oridate N, Fukuda S. Clinical and Pathological Analysis of Recurrent Inverted Papilloma. Ann Otol Rhinol Laryngol 2007; 116: 297-303.

11. Bhalla RK, Wright ED. Predicting the site of attachment of sinonasal inverted papilloma. Rhinology 2009; 47: 345-8.

12. Karkos PD, Khoo LC, Leong SC, et al. Computed tomography and/or magnetic resonance imaging for preoperative planning for inverted nasal papilloma: review of evidence. J Laryngol Otol 2009; 123: 705-9.

13. Busquets J, MD, Hwang P, MD. Endoscopic Resection of Sinonasal Inverted Papilloma: A Meta-analysis. Otolaryngol Head Neck Surg 2006; 134: 476-82.
Dirección: Jaime Osorio M.

Hospital Barros Luco Trudeau

E mail: jaime_osorio@yahoo.com 